\author{
Abualmaja KM, Siller L, Horrocks BR. \\ Metal-enhanced luminescence of silicon quantum dots: effects of \\ nanoparticles and molecular electron donors and acceptors on the \\ photofading kinetics. \\ Nanotechnology 2015, 26(14), 145704.
}

\section{Copyright:}

(C) 2015 IOP Publishing. This is the authors' manuscript of an article that was published in its final definitive form by IOP Publishing, 2015.

Link to published article:

http://dx.doi.org/10.1088/0957-4484/26/14/145704

Date deposited:

$19 / 05 / 2015$

Embargo release date:

18 March 2016

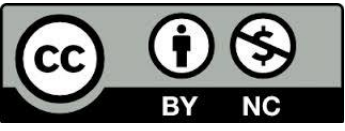

This work is licensed under a Creative Commons Attribution-NonCommercial 3.0 Unported License 


\title{
Metal-enhanced luminescence of silicon quantum dots: effects of nanoparticles and molecular electron donors \& acceptors on the photofading kinetics
}

\author{
Khamael M. Abualnaja ${ }^{1}$, Lidija Šiller ${ }^{1}$ and Benjamin R. Horrocks ${ }^{2 *}$
}

February 24, 2015

${ }^{1}$ School of Chemical Engineering and Advanced Materials, Herschel Building, Newcastle University, NE1 7RU, UK

${ }^{2}$ Chemical Nanoscience Laboratory, School of Chemistry, Bedson Building, Newcastle University, NE1 7RU, UK

\begin{abstract}
Alkyl-capped silicon quantum dots show enhanced luminescence when drop cast as films on glass slides in mixtures with $\mathrm{Ag}$ or $\mathrm{Au}$ nanoparticles or the electron donor ferrocene. Metal enhancement of quantum dot photoluminescence is known to arise from a combination of the intense near-field associated with the surface plasmon of the metal on the rate of absorption and the decrease in the lifetime of the excited state. Here we present evidence that an additional factor is also involved: electron transfer from the metal to the quantum dot. Under CW irradiation with an argon ion laser at $488 \mathrm{~nm}$, silicon quantum dots undergo a reversible photofading of the photoluminescence as the particles photoionise. A steady-state condition is established by the competition between photoionisation and electron-hole recombination. The fading of the initial photoluminescence $I_{0}$ to the steady-state value $I_{\infty}$ can be modelled by a simple first order decay with a lognormal distribution of rates, which reflects the heterogeneity of the sample. In the presence of $\mathrm{Ag}$ and $\mathrm{Au}$ nanoparticles, the modal rate constants of photofading increase by factors of up to 4 -fold and the ratio $I_{0} / I_{\infty}$ decreases by factors up to 5 -fold; this is consistent with an increase in the rate of electron-hole recombination facilitated by the metal nanoparticles acting as sources of electrons. Further support for this interpretation comes from the enhancement in photoluminescence observed in photofading experiments with films of silicon quantum dots mixed with ferrocene; this compound is a well-known one-electron donor, but shows no plasmon band which complicates the estimation of PL enhancement with Ag NPs.
\end{abstract}

\section{Introduction}

Silicon quantum dots (SiQDs) are being intensively studied because of their potential as luminescent labels $[1,2,3]$ and applications in solar energy conversion technology $[4,5]$. In particular, they may have advantages over the current technology (CdSe QDs) in applications where their lack of cytotoxicity $[6,7,8]$ and smaller overall size are important factors [2]. The indirect nature of the bulk Si bandgap and the relatively long (of order $10 \mu \mathrm{s}$ ) radiative lifetimes observed in some preparations $[9,10,11,12,13,14,15]$ has been considered a disadvantage in applications where the photoluminescence (PL) of each particle is observed for only a short length of time. An example of such an experiment might be a confocal investigation of individual moving particles. In steady-state experiments, the room-temperature quantum yield of the PL has been observed to be of order 1-10\% for uncapped SiQDs obtained from porous silicon [16], 10-20\% in alkyl-capped SiQDs [17] and close to $100 \%$ in some individual particles [15]; therefore SiQD labels are already sufficiently bright for some interesting bioimaging experiments [18, 19, 20, 21].

*b.r.horrocks@ncl.ac.uk 
Nevertheless, there have been several attempts to improve the brightness of SiQDs by the technique of metalenhanced luminescence in which SiQDs are placed in close proximity to metal nanostructures $(\mathrm{Ag}, \mathrm{Au})$ which have a strong surface plasmon resonance in the visible spectrum [22, 23, 24].

Metal-enhanced luminescence of molecular fluorophores [25], quantum wells/diodes [26, 27, 28] and quantum dots $[4,29,30,31,32,33,34]$, including silicon quantum dots [35, 36, 37, 38, 40,41, 42, 43, 44, 45, 46, 47, 48, 49], has been observed. The mechanism of the effect is attributed to coupling of the intense local electric field of the surface plasmon resonance to the absorption and emission transitions $[25,35,50]$. The surface plasmon resonance of $\mathrm{Ag}$ and $\mathrm{Au}$ nanoparticles gives rise to very large absorption coefficients and this alone can increase the brightness of the label through the increase in the rate of absorption. In addition, it has also been shown that the radiative decay rate of the emitter is increased because of the effect of the coupling on the optical density of states [39]. We have previously observed large enhancements (up to $\times 15$ ) for SiQDs co-encapsulated with AuNPs in polymer latices [40]; in part this is because, in the absence of AuNPs, the weak absorption of the SiQDs at the laser wavelength (488 $\mathrm{nm}$ ) and scattering by the polymer produces a low PL intensity. Even larger enhancements have been reported: $\times 23$ for CdSe QDs [31], $\times 60$ for SiQDs in SiN with Ag NPs [41] and ×50 AgNP/SiQD mixtures [42].

To-date investigations of metal-enhanced PL at SiQDs have focused on the steady-state spectra [43, 44, 45], but some report also measurements of the radiative lifetime [39, 46, 47, 48, 49] and the expected polarisation-selectivity of the enhancement has been observed in the presence of $\mathrm{Ag}$ nanorods [38]. Lakowicz and coworkers have also reported on the 'quenching' of the blinking of CdTe QDs in the presence of silver island films [51]. This result is of particular interest because it suggests that additional mechanisms involving the phenomena responsible for blinking or photofading, distinct from plasmon enhancement of absorption and emission rates, may increase the enhancement effect measured in ensembles. These effects are explored in this paper using mixtures of SiQDs and metal nanoparticles.

Blinking or luminescence intermittency of QDs has been suggested to occur via a photoionisation process in which an electron is ejected from the particle into the surroundings, leaving a hole, which may be trapped near the particle surface $[52,53,54]$. The charged particle appears dark because excitons produced by subsequent absorption of another photon have an efficient non-radiative decay channel involving energy transfer to the hole. This simple picture of luminescence blinking in QDs does not account for all the experimental data and alternative, but related models have been proposed $[55,56,57,58,59,60,61]$. There is direct evidence for particle charging in CdSe by electric force microscopy under irradiation [62], from the electrochemical control of blinking in core-shell CdSe/CdS [59] and in drop-cast films of SiQDs we have observed directly the photocurrents produced during irradiation at $488 \mathrm{~nm}$ in a confocal microscope [63]. In the case of SiQDs, the long radiative lifetime makes the Auger ionization and quenching processes efficient [54,64]. In the confocal-photocurrent experiment, the blinking appears as a reversible photofading of the luminescence under $\mathrm{CW}$ irradiation because we are observing the evolution of the populations of bright and dark states in the focal volume [63]. We have previously shown that alkyl-capped SiNCs are stable towards photooxidation, except under aggressive conditions [65]. The stability of alkyl-capped SiQDs towards oxidation or other irreversible chemical processes makes them a good system in which to study the kinetics of the photofading process.

In this report we report investigations of the enhanced PL observed in drop-cast films of SiQDs mixed with either noble metal nanoparticles $(\mathrm{Ag}, \mathrm{Au})$ or one-electron donors/acceptors (ferrocene/ $\mathrm{FeCl}_{3}$ ). These experiments are designed to test the hypothesis that electron injection into the SiQDs under CW irradiation is responsible for the diminution of the photofading and contributes to the overall PL enhancement. Charge transfer effects have been reported to enhance PL in QDs, however these phenomena are different to those reported here and relate to dark interactions between metal and semiconductor, e.g., the case of $\mathrm{ZnO} / \mathrm{Ag}$ [66]. First we describe the model used to analyse the experimental photofading data (section 2), then we describe the experimental methods, including $\mathrm{SiQD}$ and nanoparticle preparation (section 3). In the results we discuss the changes in the initial and steady-state PL spectra under irradiation with the $488 \mathrm{~nm}$ line of an argon ion laser (section 4.1), then the reversibility of the photofading process (section 4.2) and finally we fit a distributed kinetics model for the fading to the decay of the PL under CW irradiation (section 4.3). A comparison of the photofading curves in the presence and absence of $\mathrm{Ag}$ \& Au NPs or the one-electron donor ferrocene provides evidence for the injection of electrons into the SiQDs under irradiation and the role of such electron transfer processes in the PL enhancement. 


\section{Model}

The photofading of SiQDs under CW irradiation has been treated previously [63] by an amended version of the original random telegraphing model of Efros \& Rosen [53]. Evidence for this model comes from three observations: (i) upon cessation of the irradiation, electron-hole recombination occurs in the dark and the photoluminescence is regained when irradiation recommences; (ii) we have previously detected the photocurrent produced under CW irradiation of the SiQDs [63] and (iii) the initial and steady-state spectra are identical, except for a scale factor. Here we briefly describe the model and state those relationships which are of use in analysing the present data on metal-enhanced luminescence.

$$
\begin{gathered}
|0\rangle+n \hbar \omega \stackrel{W}{\rightleftharpoons}|1\rangle \stackrel{k_{a}}{\longrightarrow}|2\rangle \stackrel{k_{e h}}{\longrightarrow}|1\rangle \\
|1\rangle \stackrel{k_{v r}}{\longrightarrow}\left|1^{\prime}\right\rangle \stackrel{k_{\text {rad }}}{\longrightarrow}|0\rangle
\end{gathered}
$$

SiQDs are excited from their ground state $|0\rangle$ to an excited state $|1\rangle$ by absorption of 1 or more photons at a rate $W$. Autoionization of the particles with a rate constant (inverse lifetime) of $k_{a}$ produces a charged state $|2\rangle$. The particles may then discharge by electron-hole recombination with a rate constant $k_{e h}$. The excited state $|1\rangle$ may also undergo vibrational relaxation $k_{v r}$ and radiative decay $k_{r a d}$ to produce the measured photoluminescence. The excited state $|1\rangle$ is therefore 'bright' whereas the charged state $|2\rangle$ is expected to be 'dark' because of a rapid non-radiative decay pathway for excited states formed upon absorption of photons by state $|2\rangle$. For simplicity, this pathway is not shown explicitly in the model because it is assumed that the internal quantum yield for 'dark' particles in state $|2\rangle$ is zero. The measured radiative lifetime is $k_{\text {rad }} \simeq 20 \mu \mathrm{s}$ (modal value) [9]; the rates of absorption and emission are therefore much faster than the rates of the ionisation (of order $100 \mathrm{~ms}$ ) and recombination processes (minutes) and the applied light field rapidly establishes a quasi-equilibrium between the ground and excited states $|0\rangle$ and $|1\rangle$. The kinetic equations describing the photofading can be written solely in terms of the ionisation $\left(k_{a}=T_{a}^{-1}\right)$ and recombination $\left(k_{e h}=T_{e h}^{-1}\right)$ rates [63]:

$$
\frac{\mathrm{d} n_{1}}{\mathrm{~d} t}=-\frac{n_{1}}{T_{a}}+\frac{n_{2}}{T_{e h}}
$$

where $n_{1}$ and $n_{2}$ are the populations of the neutral excited state and the dark charged state respectively. The population of $|1\rangle$ and therefore also the PL intensity $I(t)$ decays exponentially with an overall lifetime $\tau$ or rate constant $k$ :

$$
\tau^{-1}=\frac{1}{T_{a}}+\frac{1}{f[I(\lambda) ; \lambda] T_{e h}} \text { or } k=k_{a}+\frac{k_{e h}}{f}
$$

where $\tau^{-1}=k$ and in the limit $k t \rightarrow \infty$ we showed that [63] :

$$
\frac{T_{a}}{f[I(\lambda) ; \lambda] T_{e h}}=\frac{I_{\infty}}{I_{0}-I_{\infty}}
$$

$f[I(\lambda) ; \lambda]$ is the ratio of excited states $|1\rangle$ to ground states $|0\rangle$ at long times and is an increasing function of intensity, $I$, and a decreasing function of wavelength, $\lambda$, as a result of the form of the absorption spectrum of SiQDs [67]. In equation 5 the initial, $I_{0}$, and steady-state, $I_{\infty}$, intensities are defined as the integrals of the intensity over the spectrum of the emitted light excluding elastically-scattered light. The quantity $f$ is not easy to measure experimentally because it would require an absolute measure of the light emitted from the focal volume as well as that collected by the objective. However, it is clear from equations (4) and (5) that the rate constant $k$ extracted from the photofading curves is related to both $k_{a}$ and $k_{e h}$ and that the steady-state PL intensity is proportional to $k_{e h} / k_{a}$. We have previously shown that it is possible to estimate $f$ and make a quantitative analysis of $k_{a}$ and $k_{e h}$ as long as $k_{a} \gg k_{e h}$ [63]. In the case of metal-enhanced PL, we find that $k_{e h}$ increases and therefore a separate determination of $k_{a}$ and $k_{e h}$ is subject to large uncertainties. Nevertheless it is clear that the observed rate constant $k$ extracted from the photofading curves increases if $k_{e h}$ increases. It is also clear that the steady-state PL intensity increases as $k_{e h}$ increases and the ratio $I_{0} / I_{\infty}$ decreases. 


\section{Experimental}

\subsection{Materials}

Silver nitrate, sodium dodecyl sulfate (SDS), gold (III) chloride trihydrate $\left(\mathrm{HAuCl}_{4} .3 \mathrm{H}_{2} 0\right)$, sodium citrate, ferrocene (Fc) and iron (III) chloride $\left(\mathrm{FeCl}_{3}\right)$ were purchased from Sigma-Aldrich. All chemicals were used without further purification. Deionised water of nominal resistivity $18 \mathrm{M} \Omega \mathrm{cm}$ from a Nanopure ${ }^{T M}$ purification system (Barnstead) was used in all the experiments. Dry toluene was obtained by distillation over sodium metal.

\subsection{Preparation of alkyl SiQDs}

Alkyl-capped SiQDs $\left(\mathrm{C}_{11}=\right.$ undecyl $)$ were prepared by the method we have previously used and which have been extensively characterised for size, composition and spectroscopic properties [17,65, 67, 68, 69]. In summary, the particle size has been estimated at about $2.5 \mathrm{~nm}$ for the crystalline $\mathrm{Si}$ core and $5 \mathrm{~nm}$ overall including the $\mathrm{C}_{11}$ alkyl capping monolayer [69]. These estimates come from TEM (Si core), AFM (core+capping layer) and Scherrer analysis of the linewidth in X-ray diffraction powder patterns, which also demonstrates the crystallinity. The transient PL from alkyl-capped SiQDs has been reported previously and compared to oxide-coated SiQDs; both types of QD show similar behaviour [68]. The increased stability of the alkyl-capped SiQDs has been demonstrated in aqueous suspension [17] and, when dry, under irradiation [65, 63].

The starting material is a silicon chip $1 \times 1 \mathrm{~cm}^{2}$ which was obtained by cutting a silicon wafer (boron-doped p$\mathrm{Si}<100>, 10 \Omega \mathrm{cm}$ resistivity, PI-KEM, Tamworth, UK) using a diamond scribe. The chip was mounted in an electrochemical cell machined from polytetrafluoroethylene (PTFE). The cell compartment was circular in crosssection with an internal diameter of $1 \mathrm{~cm}$ and a Viton ${ }^{\mathrm{TM}} \mathrm{O}$-ring at the base to make a seal against the silicon chip. Electrical contact to the back of the chip was provided by a $\mathrm{Cu}$ foil. An electrolyte comprising ethanol and $48 \%$ hydrofluoric acid HF (1:1 v/v EtOH:HF) was added to the cell (volume $\simeq 2 \mathrm{~mL})$. In order to improve the uniformity of the current distribution, a piece of tungsten wire coiled into a loop was used as a counter electrode. The chip was anodically etched under galvanostatic conditions at $400 \mathrm{~mA}$ for 5 minutes $\left(500 \mathrm{~mA} \mathrm{~cm}^{-2}\right)$. After etching, the porous silicon chip was rinsed with water and blown dry using nitrogen gas. The dry porous silicon chips were observed to luminesce an orange colour under UV irradiation $(\lambda=365 \mathrm{~nm})$.

The porous silicon chips were immediately placed in $25 \mathrm{~mL}$ of dry toluene solution which contained $0.4 \mathrm{~mL} \mathrm{1-}$ undecene and refluxed for $4 \mathrm{~h}$. The reflux was carried out in a Schlenk flask on a glass vacuum line with greasefree Young's taps and a flow of nitrogen gas was maintained in order to reduce the oxygen concentration in the solution. Under an UV Lamp $(\lambda=365 \mathrm{~nm})$ an orange colour luminescence was emitted by the resulting pale-yellow suspension. The solvent and unreacted alkene were removed from this luminescent solution under reduced pressure. The remaining product was a waxy pale yellow solid which luminesced orange under a UV lamp $(\lambda=365 \mathrm{~nm})$ and is soluble in nonpolar solvents such as tetrahydrofuran (THF) and dichloromethane. Typically about $100 \mu \mathrm{g}$ of alkyl SiQDs were formed per chip.

\subsection{Preparation of silver and gold nanoparticles}

AgNPs were prepared according to the procedure described by Bhaduri et al. [70]. We chose this procedure, rather than direct chemical reduction of $\mathrm{AgNO}_{3}(\mathrm{aq})$, for two reasons: (i) choosing concentrations of the surfactant above or below the critical micelle concentration allowed us to adjust the particle size and (ii) the absence of deliberately added reducing agents means there is less chance of confounding effects in the photofading studies arising from electron transfer between traces of reducing agent and the SiQDs.

AgNPs were synthesised from $\mathrm{AgNO}_{3}$ and sodium dodecyl sulfate (SDS) which were each dissolved in $100 \mathrm{~mL}$ of distilled water separately. The silver nitrate solution was added to SDS solution in a $200 \mathrm{~mL}$ sealed sample vial and exposed to natural sunlight. The exposure time for the reaction was 1 hour and the measured sunlight intensities were $\simeq 50 \mathrm{~mW} \mathrm{~cm}{ }^{-2}$. The temperature of the vial was maintained at $25^{\circ} \mathrm{C}$ throughout exposure to sunlight using a water bath (BS5, Fisher Scientific). After synthesis, the vials of silver nanoparticles were stored in the dark. Two kinds of AgNP samples were prepared which we denote AgNPs (1mM) and AgNPs (10mM). For AgNPs (10mM) the concentration of $\mathrm{AgNO}_{3}$ was $10 \mathrm{mM}$ and that of SDS was $50 \mathrm{mM}$ (SDS to $\mathrm{AgNO}_{3}$ molar ration 5:1). For AgNPs $(1 \mathrm{mM})$ the concentrations of $\mathrm{AgNO}_{3}$ and SDS were $1 \mathrm{mM}$ and $5 \mathrm{mM}$ (SDS to $\mathrm{AgNO}_{3}$ molar ratio fixed at 5:1). 
The different preparations have substantially different AgNP particle sizes because the critical micelle concentration of SDS is about $8 \mathrm{mM}$ under the reaction conditions. The average diameter of AgNPs $(10 \mathrm{mM})$ was $30 \mathrm{~nm}$ and of AgNPs (1mM) was $\simeq 100 \mathrm{~nm}$ by HRTEM [70]. Optical absorption spectra of the AgNPs (1mM) and AgNPs $(10 \mathrm{mM})$ samples showed $\lambda_{\max } \simeq 400 \mathrm{~nm}$ as previously reported [70].

AuNPs were synthesised according to the standard citrate method [71] with a final Au concentration of $0.41 \mathrm{mM}$. Briefly, an aqueous solution of gold (III) chloride trihydrate $\left(\mathrm{HAuCl}_{4} \cdot 3 \mathrm{H}_{2} 0\right)(0.016 \mathrm{~g}$ in $66 \mathrm{~mL}$ water $)$ was brought to reflux and then sodium citrate $(32.8 \mathrm{mg}, 1.1 \mathrm{mmol}$ in $34 \mathrm{~mL}$ water) was injected. The solution was allowed to reflux for a further $30 \mathrm{~min}$ at which point it was a dark red colour. The solution was allowed to cool and stored at room temperature. This protocol produces AuNPs with a diameter of about $20 \mathrm{~nm}$ and optical spectra similar to those reported in Ref. [72] (see Supporting information for TEM and optical absorption data).

\subsection{Confocal photoluminescence microspectroscopy}

A confocal microscope (WiTec Confocal Raman Microscope model CRM200, Ulm, Germany) at Newcastle University was used to report the temporal and spectral dependence of the intensity of photoluminescence emitted by the investigated samples under CW laser irradiation. An argon ion laser (Melles-Griot) with output power $35 \mathrm{~mW}$ at a wavelength of $488 \mathrm{~nm}$ was used as the excitation source. Emitted light was transmitted through a Raman edge filter to attenuate the elastically scattered component and collected by a multimode optical fiber which also served as the confocal pinhole. The light was then dispersed on a grating (150 lines $\left.\mathrm{mm}^{-1}\right)$ and detected using a Peltier-cooled CCD camera. The intensity reported is the integral of the spectrum over the range of wavenumbers from 200 and $7000 \mathrm{~cm}^{-1}$ with respect to the excitation light. Spectra were collected every second upon opening the laser shutter mechanically and recorded for periods up to $5 \mathrm{~min}$; the sample was under continuous, not pulsed, irradiation during this time and this is denoted $\mathrm{CW}$ in the text. The photofading curves are plots of the integral of the spectrum against recording time. Replicate measurements were performed by moving the microscope onto another area of the sample in order to collect a representative dataset.

\subsection{Preparation of samples for confocal microscopy}

Samples of SiQDs were drop-cast onto glass coverslips from THF solution (200 $\mu \mathrm{g}$ SiQDs in $100 \mu \mathrm{L}$ of THF) using a micropipette (Eppendorf) and allowed to dry in air overnight and kept in sealed Petri dishes to avoid contamination. Glass coverslips were chosen because they were observed to have no luminescence background and only very weak, broad Raman features themselves.

Mixtures of AgNPs and SiQDs were prepared by adding $100 \mu \mathrm{L}$ of aqueous AgNPs suspension to $100 \mu \mathrm{L}$ of a THF solution of SiQDs $(200 \mu \mathrm{g})$ in a centrifuge tube $(1.5 \mathrm{~mL}$, Eppendorf). The mixture was then sonicated for 10 minutes (Hilsonic). Although SiQDs are insoluble in water, they do form a kinetically-stable lyophobic sol when a THF solution is mixed with water [17]. The mixture $(100 \mu \mathrm{L})$ was then drop-coated on a glass coverslip using a micropipette and the samples were air dried overnight and kept in sealed Petri dishes. Using the previously-reported SiQD \& AgNP size estimates [69, 70] and the $\mathrm{Ag}^{+}$concentrations of the preparation we estimate the volume ratio of the SiQDs : Ag NPs in the samples dried on the glass coverslips studied to be $240: 1$ for the $\operatorname{SiQD}+\mathrm{Ag}(1 \mathrm{mM})$ sample and 24:1 for the $\mathrm{SiQD}+\mathrm{Ag}(10 \mathrm{mM})$ sample. In a few control experiments, mixtures of AgNPs and silicon nanocrystals (SiNCs) were prepared in the same manner; the SiNCs were obtained by sonication of powdered silicon in aqueous suspension (Model VCX750 Ultrasonic processor + microtip, Sonics \& Materials Inc.) and were observed by electron microscopy (TEM \& SEM) to be about $65 \mathrm{~nm}$ in diameter and crystalline.

$\mathrm{SiQD} / \mathrm{AuNPs}$ samples were also prepared by adding $100 \mu \mathrm{L}$ of a THF solution of SiQDs (200 $\mu \mathrm{g})$ to $100 \mu \mathrm{L}$ of AuNPs $(0.41 \mathrm{mM} \mathrm{Au})$ in a $1.5 \mathrm{~mL}$ centrifuge tube. A similar procedure was used to prepare the $\mathrm{SiQD} / \mathrm{FeCl}_{3}$ samples, in which a $1 \mathrm{mM}$ aqueous solution of $\mathrm{FeCl}_{3}$ replaced the $\mathrm{AgNP}$ suspension. In the case of the $\mathrm{SiQD} /$ ferrocene samples, SiQDs and ferrocene $(1 \mathrm{mM})$ were both prepared as solutions in dichloromethane (in which they are both soluble), mixed 1:1 and $100 \mu \mathrm{L}$ drop cast as before. A similar estimate to that above for the volume ratio SiQD:AuNP gives a value of $600: 1$.

The following notation is used throughout for the different samples: ' $\mathrm{C}_{11}$-SiQDs' denotes alkyl-capped silicon quantum dots alone, ' $+\operatorname{AgNPs}(1 \mathrm{mM})$ ' and ' $+\operatorname{AgNPs}(10 \mathrm{mM})$ ' denote mixtures of SiQDs and the AgNPs prepared at different $\mathrm{AgNO}_{3}$ concentrations, ' + AuNPs', ' $+\mathrm{Fc}$ ' and ' $+\mathrm{FeCl}_{3}$ ' denote the corresponding mixtures with AuNPs, ferrocene and iron (III) chloride respectively. 


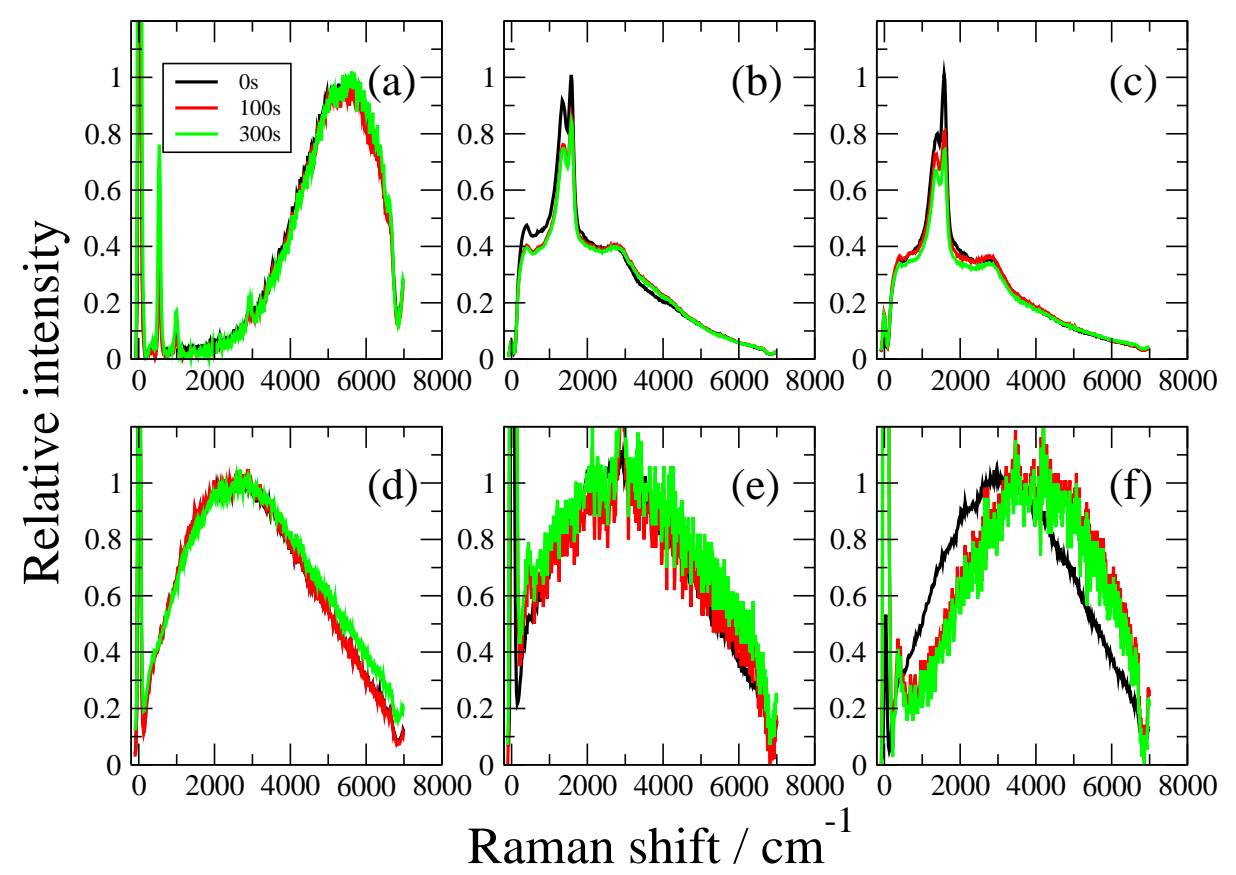

Figure 1: Raman-luminescence spectra $\left(\lambda_{e x}=488 \mathrm{~nm}\right)$ for various SiQD samples in the presence of AgNPs, AuNPs and ferrocene or $\mathrm{FeCl}_{3}$. For each panel, the spectra at the commencement of irradiation (black curves, $\mathrm{t}=0 \mathrm{~s}$ ) and after various times of irradiation (red curves, $\mathrm{t}=100 \mathrm{~s}$; green curves, $\mathrm{t}=300 \mathrm{~s}$ ) are shown. All the curves are normalised by the peak intensity to facilitate comparison of changes in shape with irradiation time. (a) $\mathrm{C}_{11}-\mathrm{SiQDs}_{\mathrm{Q}}$; (b) $+\operatorname{AgNPs}(1 \mathrm{mM})$; (c) $+\operatorname{AgNPs}(10 \mathrm{mM})$; (d) + AuNPs; (e) $+\mathrm{Fc}$; (f) $+\mathrm{FeCl}_{3}$.

\section{Results and Discussion}

\subsection{Luminescence spectra of noble metal NP-SiQD mixtures}

Figure 1 shows the PL spectra at various times after irradiation for SiQD films and films containing mixtures of SiQDs and Au or Ag NPs. The black, red and green curves are the initial spectra and after 100s and 300s irradiation respectively. The spectra for drop-cast films of SiQDs alone show the characteristic features of SiQD PL - a large Stokes' shift between the excitation line at $488 \mathrm{~nm}$ and the emission peak at a Raman shift $\simeq 5000 \mathrm{~cm}^{-1}$ or $650 \mathrm{~nm}$ in terms of wavelength. The Raman band of the $\mathrm{Si}$ core is also clearly visible at $\simeq 515 \mathrm{~cm}^{-1}$ and the second order feature at $\simeq 960 \mathrm{~cm}^{-1}$. The $\mathrm{C}-\mathrm{H}$ stretching modes of the $\mathrm{C}_{11}$ alkyl capping are present as a weak band just under $3000 \mathrm{~cm}^{-1}$. In bulk Si, the first order Raman band is at $520.7 \mathrm{~cm}^{-1}$ and the redshift in the energy of the vibration modes of SiQDs is a known effect of size quantisation on the optical phonons [73]. Together, these Raman \& PL features confirm the current samples are consistent with our previous reports. The SiQD/AgNP samples also show intense features due to Raman scattering at $1358 \mathrm{~cm}^{-1}$ and $1585 \mathrm{~cm}^{-1}$. These two bands are assigned to $\mathrm{CH}_{2}$ bending/deformation vibration modes of the dodecyl sulfate anions used to prepare the AgNPs and of the undecyl capping of the SiQDs. Other Raman bands from molecular species employed in the preparation are also visible in figure 1e; the band at $\simeq 3000 \mathrm{~cm}^{-1}$ is assigned to the $\mathrm{sp}^{2} \mathrm{C}-\mathrm{H}$ stretching modes of ferrocene [74].

It is clear from table 1 that there is a substantial increase in overall spectral intensity in the presence of metal NPs. However, especially in the case of Ag NPs, there is a large contribution from the SERS effect for the C-H bending vibrations enhanced by the AgNPs described above. This effect comprises two contributions: the sharp SERS bands and the broad SERS background. In the calculation of PL enhancement, we subtract the intensities of both these features as discussed below. Figure (2) shows examples of Raman-luminescence spectra of SiQDs + AgNPs or SiQDs + AuNPs and of AgNPs and Au NPs alone (no Si particles present). 


\begin{tabular}{|c|c|c|c|c|c|c|}
\hline & $\mathrm{C}_{11}-\mathrm{SiQDs}$ & $+\operatorname{AgNPs}(1 \mathrm{mM})$ & $+\operatorname{AgNPs}(10 \mathrm{mM})$ & + AuNPs & + Ferrocene & $+\mathrm{FeCl}_{3}$ \\
\hline Replicates: & $(n=12)$ & $(n=17)$ & $(n=15)$ & $(n=9)$ & $(n=10)$ & $(n=11)$ \\
\hline $\mathrm{PL}_{\text {enhancement }}{ }^{1}$ & 1.0 & $14 \pm 2$ & $39 \pm 8$ & $3.4 \pm 0.4$ & $5.1 \pm 0.5$ & $0.7 \pm 0.2$ \\
\hline $\mathrm{PL}_{\text {enhancement }}{ }^{2}$ & 1.0 & 9 & 30 & $\mathrm{n} / \mathrm{a}$ & $\mathrm{n} / \mathrm{a}$ & $\mathrm{n} / \mathrm{a}$ \\
\hline
\end{tabular}

Table 1: Photoluminescence intensity for SiQDs under irradiation at $488 \mathrm{~nm}$ from an argon ion laser and in the presence of noble metal nanoparticles and one-electron donors/acceptors. The intensity reported is $\int_{0}^{300} I(t) \mathrm{d} t$, i.e., the spectral intensity $I$ integrated over the full range of the wavenumbers (excluding elastically scattered light) and over a period of $5 \mathrm{~min}$ of irradiation. ${ }^{1}$ These enhancement factors have been corrected to exclude the SERS bands visible in + AgNPs samples. ${ }^{2}$ These enhancement factors have additionally been corrected, in an approximate manner discussed in the text, for the SERS background in + AgNPs samples.
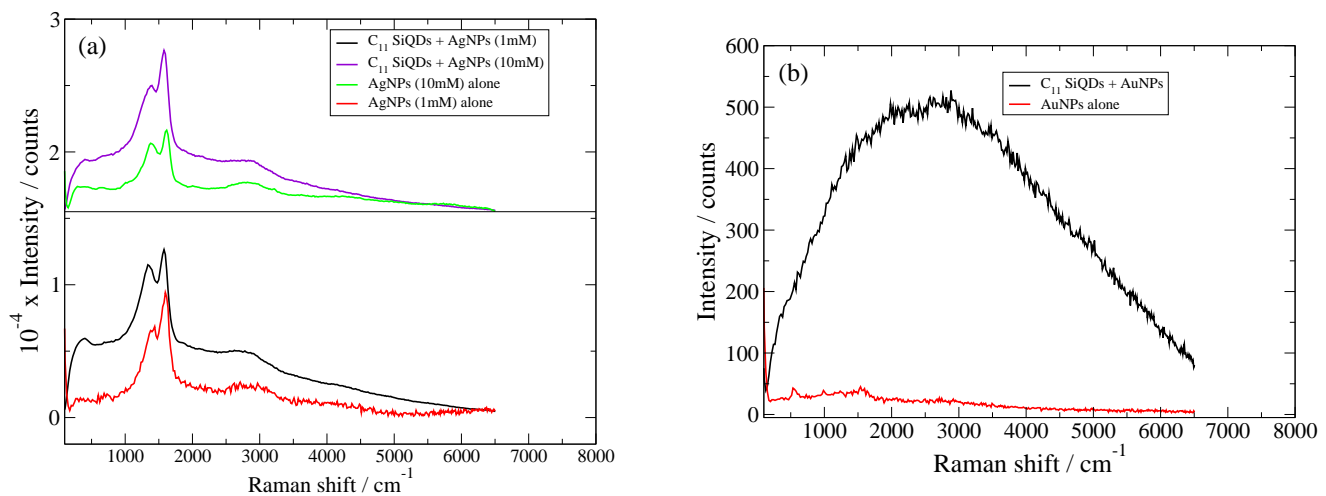

Figure 2: Raman-luminescence spectra $\left(\lambda_{e x}=488 \mathrm{~nm}\right.$ ) for various SiQD samples in the presence of AgNPs, AuNPs and of AgNPs and AuNPs in the absence of SiQDs. The integration time was 1s in every case. (a) $\mathrm{SiQDs}+\mathrm{Ag}(1 \mathrm{mM})$ and SiQDs $+\mathrm{Ag}(10 \mathrm{mM})$. The Raman spectra for $\mathrm{Ag}(1 \mathrm{mM})$ and $\mathrm{Ag}(10 \mathrm{mM})$ alone are also shown. The spectra of the $\mathrm{Ag}(10 \mathrm{mM})$ samples with and without SiQDs have been offset on the y-axis for clarity. (b) SiQDs+AuNPs and AuNPs Raman-luminescence spectra. 
In order to estimate the PL enhancement, we compared the integrated intensity of the spectra of a given sample to that for SiQDs alone. The intensities used in the enhancement calculation were integrated over the spectral range 200 and $7000 \mathrm{~cm}^{-1}$ (to avoid the contribution from elastically scattered light at $0 \mathrm{~cm}^{-1}$ ) and also integrated over a time of $300 \mathrm{~s}$ of irradiation sufficient to attain a steady-state (such values of $\int_{0}^{300} I \mathrm{~d} t$ are given in tables $2 \& 3$ below). The values in table 1 show the ratios of integrated intensity of the various mixtures against the pure SiQD spectra, but with two corrections applied to the spectra of samples containing AgNPs. In the first calculation (PL enhancement ${ }^{1}$ ), the intensity of the $\mathrm{CH}_{2}$ Raman bands has been subtracted from the integral for the samples containing AgNPs. The $\mathrm{CH}_{2}$ bending/deformation Raman bands (centred around $1500 \mathrm{~cm}^{-1}$ in figures $1 \mathrm{~b}$,c and 2a) are sufficiently large to account for $20 \%$ of the integrated intensity in those spectra, but after correction for this factor the enhancements are still very substantial - up to a factor of $\times 30$ in the case of the AgNP (10mM) samples. This is comparable with enhancement ratios of $\times 60$ [41] and $\times 50$ [42] in other AgNP/SiQD systems. The second correction (PL enhancement ${ }^{2}$ ) in table 1 includes the first and in addition corrects for the SERS background [75]. This is the broad background always associated with intense SERS peaks and has typically not been accounted for in reports of large enhancement factors in the $\mathrm{AgNP} / \mathrm{SiQD}$ system [41], however it may be significant. An estimate of this correction was obtained from control experiments in which samples of nanocrystalline silicon powder (SiNCs: mean particle diameter $\simeq 65 \mathrm{~nm} \gg$ Bohr radius in $\mathrm{Si}$ ) were mixed with $\mathrm{AgNPs}(1 \mathrm{mM})$ and AgNPs $(10 \mathrm{mM})$ in the same manner as for SiQDs. The PL spectra of these mixtures showed similar features to those of AgNPs discussed above, but with an integrated intensity of $\simeq 30 \%$ of the $\mathrm{C}_{11}$-SiQDs $+\mathrm{AgNPs}(1 \mathrm{mM})$ sample and $\simeq 18 \%$ of the $\mathrm{C}_{11^{-}}$ SiQDs + AgNPs $(10 \mathrm{mM})$ sample respectively. We interpret these spectra mainly in terms of the SERS bands and the SERS background - they do not show the same photofading $\left(I_{0} / I_{\infty} \simeq 1.1 \pm 0.3 ; n=10\right)$ as SiQDs - and therefore we estimate that the contribution of the SERS background to the enhancement factors in table 1 is about $30 \%$ of the $\mathrm{C}_{11}-\mathrm{SiQDs}+\mathrm{AgNPs}(1 \mathrm{mM})$ sample and $18 \%$ of the $\mathrm{C}_{11}-\mathrm{SiQDs}+\mathrm{AgNPs}(10 \mathrm{mM})$ sample. The PL enhancement in the presence of AuNPs of similar size (mean diameter $=20 \mathrm{~nm}$ ) is an order of magnitude lower. We attribute this to the unfavourable longer wavelength of the plasmon band in AuNPs ( $520 \mathrm{~nm}$, see Supporting information) compared to AgNPs ( $\simeq 400 \mathrm{~nm}[70])$; the optimal situation for SERS, and likely for metal-enhanced PL also, is for the the incident light to be on the low energy side of the plasmon resonance [76]. However, we find that because the SERS effects are much smaller for the AuNPs, the final estimates of the PL enhancement did not require any correction for Raman spectral features.

The most obvious difference between the spectra in the presence of AgNPs and their absence is the very large blueshift $\left(\simeq 3500 \mathrm{~cm}^{-1}\right)$ of the emission maximum. The coupling of the plasmon resonance to excited states of the SiQDs will be more effective for those states near the laser energy, therefore we suggest that the observed PL shift corresponds to emission from states at energies higher than those responsible for the usual $650 \mathrm{~nm}$ emission band of the pure SiQDs. For both SiQD/AgNP and SiNC/AgNP samples, the position and shape of the spectral features are dominated by the optical properties of the AgNPs and difficult to distinguish from SERS background effects. The blueshift is less for the AuNPs (although still large $\simeq 2500 \mathrm{~cm}^{-1}$ ), because the plasmon resonance is at lower energy $(\lambda=520 \mathrm{~nm})$ than the laser $(\lambda=488 \mathrm{~nm})$. Nevertheless, it is clear that simple mixtures of SiQDs with noble metal nanoparticles yield substantial increases in the intensity of emitted/scattered light. In the following sections, we observe in more detail the effects of these metal nanoparticles on the photofading kinetics and discuss their role as electron donors. In addition, we have obtained spectra for two molecular species (ferrocene and $\mathrm{FeCl}_{3}$ ) which can act as an electron donor and acceptor respectively, but do not possess the plasmon resonance typical of metals. Interestingly, there is also a blueshift $\left(\simeq 2000 \mathrm{~cm}^{-1}\right)$ in the PL spectrum of SiQDs in the presence of the one-electron donor ferrocene as well as a substantial $\times 5$ enhancement of the PL. This molecule is stable in the $\mathrm{Fe}(\mathrm{II})$ and $\mathrm{Fe}(\mathrm{III})$ states; ferrocene, the $\mathrm{Fe}(\mathrm{II})$ state, is orange in colour with only a very weak absorption tail in the blue region of the visible spectrum, but ferrocenium, the Fe(III) state formed upon electron loss, has a substantial absorption in the red at about $620 \mathrm{~nm}\left(\epsilon \simeq 350 \mathrm{M}^{-1} \mathrm{~cm}^{-1}\right.$ [77]). It is therefore possible that the apparent blueshift is simply a result of self-absorption of the long wavelength emitted light in the sample film. Neither ferrocene itself nor $\mathrm{FeCl}_{3}$ was observed to show any PL and their Raman spectra are much weaker than those observed in the presence of metal NPs (see Supporting information).

\subsection{Reversibility of the photofading}

Figure 1 also illustrates the evolution of the PL spectra of SiQDs and mixtures with irradiation time. In each panel, the spectra are scaled so that the maximum intensity is 1.0 irrespective of the time; this facilitates comparison of the shape of the spectra at different times of irradiation. In figure 1a the 3 curves corresponding to the initial, $t=100 \mathrm{~s}$ and $t=300 \mathrm{~s}$ spectra superimpose closely, except for the Raman bands previously discussed at 515,960 


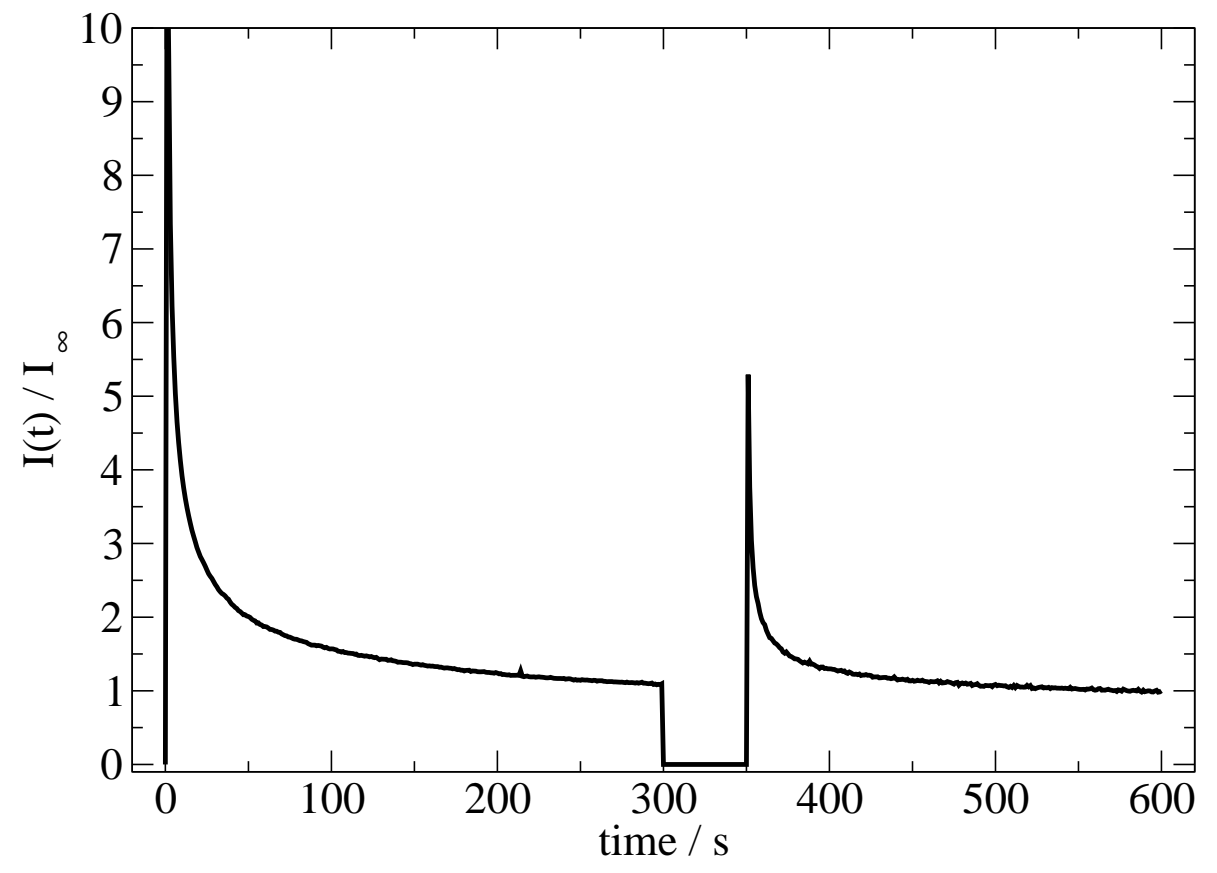

Figure 3: Intensity against time for the photofading of $\mathrm{C}_{11}-\mathrm{SiQDs}$ under irradiation with the $488 \mathrm{~nm}$ line of an argon ion laser. The intensity plotted is the integral of the spectrum at each time point over the wavenumber range $200-7000 \mathrm{~cm}^{-1}$ and normalised by the steady state value $\left(I_{\infty}\right)$. At $t=300$ s the irradiation was interrupted for $50 \mathrm{~s}$ by closing the shutter. When the shutter was reopened at $350 \mathrm{~s}, \simeq 50 \%$ of the initial PL intensity was regained.

and $\simeq 3000 \mathrm{~cm}^{-1}$ which are time-independent and therefore are apparently more intense at $t=300$ s only because of the scaling applied to the PL. The lack of any change in spectrum has been reported previously [63] and is simply interpreted in terms of the model if state $|2\rangle$ is entirely dark and the PL is entirely due to state $|1\rangle$ via the sequence of steps in equation (2). In other words, during the photofading experiment it is the population of state $|1\rangle$ that changes.

Figure 3 shows also that the PL recovers partially after a period in which the irradiation is interrupted; the reversibility of the photofading and the lack of change in the spectra support our interpretation that the fading is a physical rather than a chemical process [63]. For example, photoxidation of $\mathrm{Si}$ is likely to be completely irreversible and would also change the PL spectrum through introduction of defect states or a reduction in the the size of the $\mathrm{Si}$ core. The model we apply instead interprets these effects as a reversible charging of the SiQDs and this possibility is supported by previous observations of reversible charging/discharging in X-ray photoemission experiments [65].

In mixtures of SiQDs + AuNPs (figure 1d) or SiQDs + Fc (figure 1e) we also find PL spectra which are independent of irradiation after scaling, although a small tail appears on the low energy side of the $t=300$ s spectrum for SiQDs + AuNPs. The interpretation of the SiQDs + AgNPs spectra (figures 1b\&c) is less clear-cut because of the substantial contribution from SERS bands, however these spectra are still consistent with the simple model. The case of $\mathrm{SiQDs}+\mathrm{FeCl}_{3}$ is different; there is a substantial redshift $\left(\simeq 1000 \mathrm{~cm}^{-1}\right)$ under irradiation and this sample clearly shows a more complex behaviour than the model of equations (1) \& (2).

\subsection{Dispersed kinetics of photofading}

Figure 4 shows typical examples of photofading experiments; under CW irradiation the PL intensity decays with time. The black, red and blue curves are the initial experimental photofading data, the regression model (equation (7)) and the residual respectively. The connection between the photofading of ensembles of QDs and the statistics 


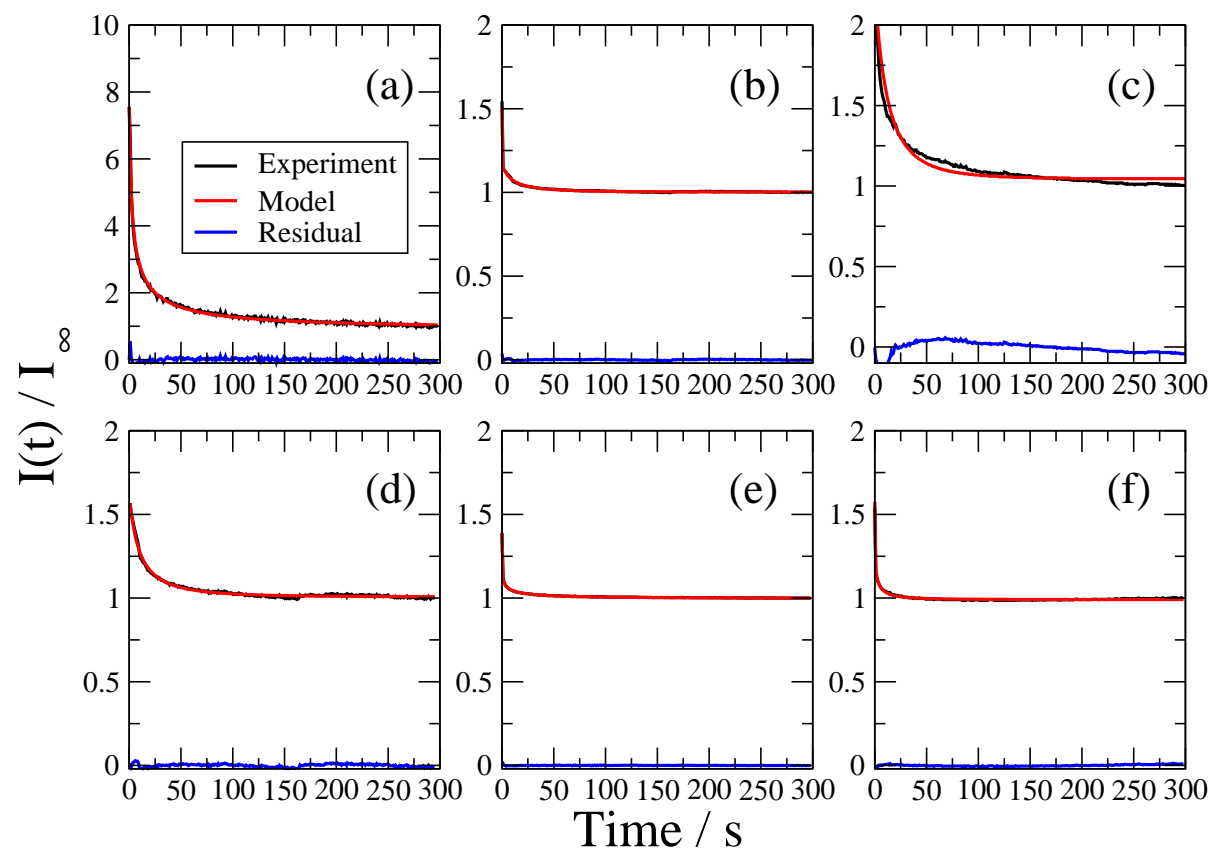

Figure 4: Photofading curves under $\mathrm{CW}$ irradiation at $\lambda_{e x}=488 \mathrm{~nm}$ for various SiQD samples in the presence of AgNPs, AuNPs and ferrocene or $\mathrm{FeCl}_{3}$. All the curves are normalised by the steady-state intensity $I_{\infty}$. The black, red and blue curves are the experimental photofading data, the regression model (equation (7)) and the residual respectively. (a) $\mathrm{C}_{11}-\operatorname{SiQDs}$; (b) $+\operatorname{AgNPs}(1 \mathrm{mM}) ;\left(\right.$ c) $+\operatorname{AgNPs}(10 \mathrm{mM}) ;(\mathrm{d})+\operatorname{AuNPs}$; (e) $+\mathrm{Fc}$; (f) $+\mathrm{FeCl}_{3}$.

of the blinking phenomenon has been explored in the case of (CdSe)ZnS core-shell particles [78]. We prefer to fit the photofading curves directly to extract the rate constant $k$ which describes the fading using a distributed kinetics model which generalises the simple first order kinetics of the random telegraphing model of QD blinking described above (section 2). In previous work, we have shown that a simple long-normal distribution of $k$, i.e., a Gaussian distribution of activation energies, provides a satisfactory regression model [63]. In this report we show that the same model also can be fitted to the photofading curves in the presence of metal NPs (Ag, $\mathrm{Au})$ and iron-based electron donors and acceptors (ferrocene, $\mathrm{FeCl}_{3}$ ).

The dispersed kinetics treatment is preferred over multi-exponential or stretched-exponential models for similar reasons to those discussed in [79]. In brief, it is natural to expect a distribution of rates for heterogeneous systems such as QDs and the parameters obtained in the regression analysis have a clearer physical meaning than the rate constants or lifetimes extracted from multiexponential fits or the parameter $\beta$ from a stretched exponential expression such as $e^{-\left(\frac{t}{\tau}\right)^{\beta}}$. In addition, the dispersed kinetics treatment has fewer floating parameters (amplitude, mode and width of the distribution) than even a double exponential fit (two amplitudes \& two lifetimes).

According to the model on which we base the analysis, each SiQD may be either bright or dark according to whether it is uncharged or has undergone photoionisation, and the concentration of bright particles is proportional to the intensity of the luminescence, $I$. A first check that the model is consistent with the experimental data can be made by comparing the spectra obtained upon initial irradiation and at longer times. Figure 1 shows that, as expected, the spectra change in intensity, but do not change shape with extended times of irradiation. It is therefore consistent to interpret them in terms of the populations of bright $(|1\rangle)$ and dark $(|2\rangle)$ states described in the theoretical model.

If $I_{0}$ is the luminescence intensity at the commencement of irradiation and $I_{\infty}$ is the steady - state intensity, the decay of $I_{0}$ to $I_{\infty}$ is modelled by a lognormal distribution of rate constants $k$ which represents the heterogeneity in the sample. The parameter $\langle k\rangle$ is the most probable value of the rate constant for the first order photofading and $\gamma$ is the spread of the Gaussian distribution of Gibbs energies for the reaction in units of RT [80]: 


$$
\Delta G=\langle\Delta G\rangle-\gamma R T x \text { and } P(x)=\pi^{-\frac{1}{2}} \exp \left(-x^{2}\right)
$$

The distribution of the rate constants is obtained from: $k=\langle k\rangle \exp (\gamma x)$ and the regression model which was employed to analyse the data is the first order decay of bright states averaged over the Gaussian distribution:

$$
I=\pi^{-\frac{1}{2}}\left(I_{0}-I_{\infty}\right) \int_{-\infty}^{\infty} \exp \left(-x^{2}\right) \exp [-\langle k\rangle t \exp (\gamma x)] d x+I_{\infty}
$$

The model was fitted to the data using the Levenberg-Marquardt method and an extended Simpson's rule using 100 integration points was used to evaluate the integral on the range $u[-1,1]$ after making the transformation $\ln u=x$ and splitting the original range of integration into $[-\infty, 0]$ and $[0, \infty]$. Four parameters were extracted from the fitting of equation 7 to the photofading curves: $I_{0}, I_{\infty},\langle k\rangle$ and $\gamma$. Tables $2 \& 3$ show the values of these parameters for six types of sample and their means and standard deviations over a number of replicate experiments. In addition, we report the time-integrated intensity over a period of $300 \mathrm{~s}$ irradiation $\int_{0}^{300} I \mathrm{~d} t$ and the ratio of the initial and steady-state intensities, $I_{0} / I_{\infty}$.

The curves in figure 4 have been normalised by $I_{\infty}$ in order to display all the data together. The residual is shown on each trace and for most of the samples, we found a good fit of equation (7) to the data, as judged by a small residual $(\simeq 1 \%)$ which showed no systematic deviations from zero. The exceptions to this were photofading curves for the $+\operatorname{AgNPs}(10 \mathrm{mM})$ samples (figure $4 \mathrm{c}$ ) in which systematic deviations were typically observed. We suggest that these are related to heating effects in the confocal volume.

\subsubsection{Effect of metal NPs on the photofading kinetics}

The presence of either Ag or AuNPs in a simple mixture with SiQDs produces a substantial enhancement of the integrated PL intensity, up to $\times 30$ in the case of SiQDs mixed with the $10 \mathrm{mM}$ AgNP preparation (mean particle size $\simeq 30 \mathrm{~nm}$ ). It is also noteworthy that the extent of photofading, measured by the ratio $I_{0} / I_{\infty}$ is substantially less in the presence of metal NPs. The normal explanation for the PL enhancement by metal NPs is in terms of the efficient coupling of the excitation light to the SiQDs via the surface plasmon resonance of the metal NPs and of the coupling of the emissive states to the NPs [25, 35, 50]. SiQDs have a relatively low absorbance at $488 \mathrm{~nm}$, but the absorption coefficient of metal NPs near the plasmon resonance is known to be very large and so long as the SiQDs are within the range of the near-field at the metal surface, the rate of absorption can be much greater. However, such an effect does not account for the observation of a smaller $I_{0} / I_{\infty}$ ratio in the presence of NPs, in fact, this ratio might be expected to increase because the rate of photoionisation depends on the number of SiQDs in an electronic excited state. If the rate of recombination does not increase proportionately, then the steady-state PL intensity will decrease and the ratio $I_{0} / I_{\infty}$ will increase. The decrease in the ratio $I_{0} / I_{\infty}$ by factors of $3-5$ in the presence of $\mathrm{Ag}$ or $\mathrm{Au}$ NPs therefore provides evidence that there is a second mechanism for the enhancement of the PL; we suggest that the metal NPs act as a source/sink for electrons and increase the rate of recombination $\left(k_{e h}|2\rangle\right)$. This effect increases the PL because state $|2\rangle$ is dark and only state $|1\rangle$ is emissive.

The metal NPs also have a substantial effect on the kinetics of the photofading; the most probable rate constant describing the decay of the initial intensity towards the steady-state increases and the width of the distribution of rates decreases. The increase in the modal value of the rate constant is quite expected on the basis of the reaction scheme (equations 1-2). In an analogous manner to the well-known behaviour of chemical reactions near equilibrium, the observed rate constant is the sum of the forward and backward rate constants. In the present case the system is not at equilibrium, but rather tends towards a steady-state at long times, nevertheless the steady-state is reached more quickly if either the rate of ionisation or of recombination increases (equation (4)). The AgNPs and, to a lesser extent, the Au NPs act as sources of electrons and can quench the charge developing on the SiQDs in the focal volume.

\subsubsection{Effect of one-electron donors \& acceptors on the photofading kinetics}

In order to explain the decrease in the ratio $I_{0} / I_{\infty}$ by factors of 3-5 in the presence of $\mathrm{Ag}$ or Au NPs, we suggest that exchange of electrons occurs between the metal NPs and the SiQDs. This is also an important factor contributing towards the PL enhancement, but cannot easily be distinguished from the usual plasmon-related effects. To test 


\begin{tabular}{|c|c|c|c|c|}
\hline & $\mathrm{C}_{11}-$ SiQDs & + AgNPs $(1 \mathrm{mM})$ & + AgNPs $(10 \mathrm{mM})$ & + AuNPs \\
\hline Replicates: & $(n=12)$ & $(n=17)$ & $(n=15)$ & $(n=9)$ \\
\hline \hline $10^{-7} \times \int_{0}^{300} I \mathrm{~d} t / \mathrm{counts}$ & $1.96 \pm 0.17$ & $34.2 \pm 0.50$ & $94.9 \pm 7.0$ & $6.62 \pm 0.30$ \\
\hline $10^{-5} \times I_{0} / \mathrm{cps}$ & $1.44 \pm 0.21$ & $16.1 \pm 0.30$ & $58.6 \pm 6.2$ & $4.44 \pm 0.28$ \\
\hline $10^{-5} \times I_{\infty} / \mathrm{cps}$ & $0.208 \pm 0.044$ & $10.7 \pm 0.20$ & $26.9 \pm 2.6$ & $2.88 \pm 0.10$ \\
\hline$\langle k\rangle / \mathrm{s}^{-1}$ & $0.125 \pm 0.007$ & $0.368 \pm 0.004$ & $0.461 \pm 0.004$ & $0.146 \pm 0.014$ \\
\hline$\gamma$ & $3.60 \pm 0.45$ & $2.35 \pm 0.55$ & $1.00 \pm 0.42$ & $2.47 \pm 0.40$ \\
\hline$I_{0} / I_{\infty}$ & $6.93 \pm 0.64$ & $1.45 \pm 0.03$ & $2.16 \pm 0.08$ & $2.14 \pm 0.14$ \\
\hline
\end{tabular}

Table 2: Kinetic data from photofading experiments on SiQDs in the presence of Ag and Au NPs.

\begin{tabular}{|c|c|c|c|}
\hline & $\mathrm{C}_{11}-\mathrm{SiQDs}$ & $+\mathrm{Fc}$ & $+\mathrm{FeCl}_{3}$ \\
\hline Replicates: & $(n=12)$ & $(n=10)$ & $(n=11)$ \\
\hline \hline $10^{-7} \times \int_{0}^{300} I \mathrm{~d} t /$ counts & $1.96 \pm 0.17$ & $10.0 \pm 0.20$ & $1.4 \pm 0.18$ \\
\hline $10^{-5} \times I_{0} / \mathrm{cps}$ & $1.44 \pm 0.21$ & $4.64 \pm 0.45$ & $4.26 \pm 0.17$ \\
\hline $10^{-5} \times I_{\infty} / \mathrm{cps}$ & $0.208 \pm 0.044$ & $3.33 \pm 0.06$ & $2.17 \pm 0.13$ \\
\hline$\langle k\rangle / \mathrm{s}^{-1}$ & $0.125 \pm 0.007$ & $0.240 \pm 0.015$ & $0.150 \pm 0.048$ \\
\hline$\gamma$ & $3.60 \pm 0.45$ & $2.82 \pm 0.11$ & $3.17 \pm 0.13$ \\
\hline$I_{0} / I_{\infty}$ & $6.93 \pm 0.64$ & $1.12 \pm 0.11$ & $2.15 \pm 0.22$ \\
\hline
\end{tabular}

Table 3: Kinetic data from photofading of SiQDs in the presence of electron donor (Fc) or an electron acceptor $\left(\mathrm{FeCl}_{3}\right)$.

this hypothesis, we prepared mixtures of SiQDs with molecular donors \& acceptors. Ferrocene is a well-known one-electron redox mediator which has rapid self-exchange kinetics and is known to be stable in both $\mathrm{Fe}(\mathrm{II})$ and $\mathrm{Fe}$ (III) oxidation states. It can therefore act as a source/sink of electrons in the same manner as metal NPs, but without the confounding effects related to the surface plasmon of metal NPs. Table 3 shows that ferrocene has a large effect on the ratio $I_{0} / I_{\infty}$ which is reduced by a factor of more than 6 compared to the pure $\mathrm{C}_{11}-\mathrm{SiQD}$ sample. Another redox species, $\mathrm{FeCl}_{3}$ also reduces this ratio, but as described in section 4.2 has a complicated effect on the PL spectrum. Ferrocene also reduces the spread of the rates $\gamma$ to a similar extent as do large AgNPs (1mM) (mean particle diameter $100 \mathrm{~nm}$ ) and AuNPs. We suggest that this is because the rate of electron injection into SiQDs is also a distributed kinetic process and that those particles for which the injection is rapid do not contribute to the photofading.

\section{Conclusions}

Under CW irradiation in a confocal microscope, alkyl-capped silicon quantum dots show a reversible photofading behaviour. This can be interpreted by the same model originally proposed to describe luminescence intermittency, i.e., 'blinking' [53]. Particles in an excited state may undergo either photoionisation or radiative decay; the charged particles produced by photoionisation are dark because they have an efficient non-radiative decay pathway in which energy is transferred from the exciton to the hole. When single particles are studied, this leads to the well-known blinking phenomenon as particles ionise and later discharge by electron-hole recombination. In an ensemble, the result is a reversible photofading as the initial photoluminescence $I_{0}$ decays to a steady-state $I_{\infty}$ controlled by the relative rates of photoionisation $k_{a}$ and recombination $k_{e h}$. The photofading data can be modelled as a simple first order decay with a lognormal distribution of rate constants and therefore characterised by three parameters; $\langle k\rangle$ the modal rate constant, $\gamma$, which measures the spread of activation free energies in units of $\mathrm{R} T$, and $I_{0} / I_{\infty}$.

Alkyl-capped silicon quantum dots show enhanced luminescence when drop cast as films on glass slides in mixtures with Ag or Au nanoparticles. Such metal-enchanced luminescence is generally explained in terms of the large electric field near the metal surface upon excitation of the plasmon resonance and an increase in the radiative decay rate owing to the effect of the plasmon on the optical density of states. In this work, we find evidence for an additional effect: the metal nanoparticles can act as a source of electrons and increase the time-integrated luminescence intensity by increasing the rate of electron-hole recombination. In the presence of $\mathrm{Ag}$ and $\mathrm{Au}$ nanoparticles, the modal rate constants $\langle k\rangle$ of the photofading process increase by factors of up to 4 -fold and the ratios $I_{0} / I_{\infty}$ decrease 
by factors up to 5 -fold; this is consistent with an increase in the rate of electron-hole recombination facilitated by the metal nanoparticles acting as sources of electrons. Further support for this interpretation comes from the enhancement in photoluminescence observed in photofading experiments with films of silicon quantum dots mixed with ferrocene; this compound is insulating and shows no plasmon band, but is a well-known one-electron donor.

\section{Acknowledgements}

KMA would like to thank Taif University and the Ministry of Higher Education, Saudi Arabia. Dr N. A. Harun is thanked for assisstance with characterisation of the AuNP samples.

\section{Glossary}

Fc - ferrocene

SiQDs - alkyl $\left(\mathrm{C}_{11}\right)$ capped silicon quantum dots

AgNPs $(1 \mathrm{mM})$ - silver nanoparticles prepared photochemically using $1 \mathrm{mM} \mathrm{AgNO}$ with mean particle diameter $\simeq$ $30 \mathrm{~nm}$

AgNPs (10mM) - silver nanoparticles prepared photochemically using $10 \mathrm{mM} \mathrm{AgNO}_{3}$ with mean particle diameter $\simeq 100 \mathrm{~nm}$

AuNPs - gold nanoparticles prepared by citrate reduction of gold (III) chloride

\section{References}

[1] Cheng X, Lowe S B, Reece P J and Gooding J J 2014 Chem. Soc. Rev. 432680

[2] O'Farrell N, Houlton A and Horrocks B R 2006 Int. J. Nanomed. 1451

[3] Shirahata N 2011 PCCP 137284

[4] Chandra S, Doran J, McCormack S J, Kennedy M and Chatten A J 2011 Solar Energy Materials and Solar Cells 98385

[5] Mendes M J, Hernández E, López E, García-Linares P, Ramiro I, Artacho I, Antolín E, Tobías I, Martí A and Luque A 2013 Nanotechnology 24345402

[6] Alsharif N H, Berger C E M, Varanasi S S, Chao Y, Horrocks B R and Datta H 2009 Small 5221

[7] Wang Q, Bao Y, Zhang X, Coxon P R, Jayasooriya U A and Chao Y 2012 Adv. Healthcare Mater. 1189

[8] Choi J, Zhang Q, Reipa V, Wang N S, Stratmeyer M E, Hitchins V M and Goering P L 2009 J. Appl. Toxicol. 2952

[9] Rostron R J, Horrocks B R and Roberts G 2009 J. Appl. Phys. 105094302

[10] Li H, Xu D, Guo G, Gui L, Tang Y, Ai X, Sun Z, Zhang X and Qin G G 2000 J. Appl. Phys. 884446

[11] Vial J C, Bsiesy A, Gaspard F, Hérino R, Ligeon M, Muller F, Romestain R and Macfarlane R M 1992 Phys. Rev. B 4514171

[12] Xie Y H, Wilson W L, Ross F M, Mucha J A, Fitzgerald E A, Macaulay J M and Harris T D 1992 J. Appl. Phys. 712403

[13] Kovalev D I, Yaroshetzkii I D, Muschik T, Petrova-Koch V and Koch F 1994 Appl. Phys. Lett. 64214

[14] Linnros J, Lalic N, Galeckas A and Grivickas V 1999 J. Appl. Phys. 866128 
[15] Credo G M, Mason M D and Buratto S K 1999 Appl. Phys. Lett. 741978

[16] Wilson W L, Szajowski P F and Brus L E 1993 Science 2621242

[17] Dickinson F M, Alsop T A, Al-Sharif N, Berger C E M, Datta H K, Šiller L, Chao Y, Tuite E M, Houlton A and Horrocks B R 2008 Analyst 1331573

[18] Li Z F and Ruckenstein E 2004 Nano Lett. 41463

[19] Erogbogbo F, Yong K T, Roy I, Hu R, Law W C, Zhao W, Ding H, Wu F, Kumar R, Swihart M T and Prasad P N 2011 ACS Nano 5413

[20] Zhong Y, Peng F, Bao F, Wang S, Ji X, Yang L, Su Y, Lee S-T and He Y 2013 J. Am. Chem. Soc. 1358350

[21] Ahire J H, Wang Q, Coxon P R, Malhotra G, Brydson R, Chen R and Chao Y 2012 ACS Appl. Mater. E6 Interfaces 43285

[22] Mulvaney P 1996 Langmuir 12788

[23] Liz-Marzan L M 2005 Langmuir 2232

[24] Rycenga M, Cobley C M, Zeng J, Li W, Moran C H, Zhang Q, Qin D and Xia Y 2011 Chem. Rev. 1113669

[25] Geddes C D and Lakowicz J R 2002 J. Fluorescence 12121

[26] Yeh D-M, Huang C-F, Chen C-Y, Lu Y-C and Yang C C 2008 Nanotechnology 19345201

[27] Huang C-W, Tseng H Y, Chen C Y, Liao C H, Hsieh C, Chen K Y, Lin H Y, Chen H S, Jung Y L, Kiang Y W and Yang C C 2011 Nanotechnology 22475201

[28] Cho C-Y, Kwon M-K, Lee S-J, Han S-H, Kang J-W, Kang S-E, Lee D-Y and Park S-J 2010 Nanotechnology 21205201

[29] Kulakovich O, Strekal N, Yaroshevich A, Maskevich S, Gaponenko S, Nabiev I, Woggon U and Artemyev M 2002 Nano Lett. 21449

[30] Shimizu K T, Woo W K, Fisher B R, Eisler H J and Bawendi M G 2002 Phys. Rev. Lett. 89117401

[31] Okamoto K, Vyawahare S and Scherer A 2006 J. Opt. Soc. Am. B 231674

[32] Ray K, Badugu R and Lakowicz J R 2006 J. Am. Chem. Soc. 1288998

[33] Fu Y, Zhang J and Lakowicz J R 2009 Chem. Commun. 313

[34] Liu B-T, Liao T-H, Tseng S and Lee M-H 2014 Appl. Surf. Sci. 292615

[35] Biteen J S, Pacifici D, Lewis N S and Atwater H A 2005 Nano Lett. 51768

[36] Biteen J S, Lewis N S and Atwater H A 2006 Appl. Phys. Lett. 88131109

[37] Biteen J S, Sweatlock L A, Mertens H, Lewis N S, Polman A and Atwater H A 2007 J. Phys. Chem. C 111 13372

[38] Mertens H, Biteen J S, Atwater H A and Polman A 2006 Nano Lett. 62622

[39] Goffard J, Gerard D, Miska P, Baudrion A-L, Deturche R and Plain J 2013 Sci. Rep. 32672

[40] Harun N A, Benning M J, Horrocks B R and Fulton D A 2013 Nanoscale 53817

[41] Nychyporuk T, Zakharko Yu, Serdiuk T, Marty O, Lemiti M and Lysenko V 2011 Nanoscale 32472

[42] Subramanian K K H "Spin coating of Silver Nanoparticles and Silicon quantum dots for enhanced down conversion efficiency" MSc thesis, Faculty of Applied Sciences (TNW) TU Delft, Netherlands, 2013

[43] Takeda E, Nakamura T, Fujii M, Miura S and Hayashi S 2006 Appl. Phys. Lett. 89101907

[44] Tang X, Wang Y, Ke W, Feng X, Huang Y and Peng J 2010 Opt. Commun. 2832754 
[45] Mochizuki Y, Fujii M, Hayashi S, Tsuruoka T and Akamatsu K 2009 J. Appl. Phys. 106013517

[46] Basu T S and Ray M 2014 J. Phys. Chem. C 1185041

[47] Ray M, Basu T S, Bandyopadhyay N R, Klie R F, Ghosh S, Rajad S O and Dasguptad A K 2014 Nanoscale 62201

[48] Dan'ko V, Michailovska K, Indutnyi I and Shepeliavyi P 2014 Nanoscale Res. Lett. 9165

[49] Kalkman J, Gersen H, Kuipers L and Polman A 2006 Phys. Rev. B 73075317

[50] Schaadt D M, Feng B and Yu E T 2005 Appl. Phys. Lett. 86063106

[51] Fu Y, Zhang J and Lakowicz J R 2007 Chem. Phys. Lett. 44796

[52] Nirmal M, Dabbousi B O, Bawendi M G, Macklin J J, Trautman J K, Harris T D and Brus L E 1996 Nature 383802

[53] Efros A L and Rosen M 1997 Phys. Rev. Lett. 781110

[54] Efros A L, Rosen M, Averboukh B, Kovalev D, Ben-Chorin M and Koch F 1997 Phys. Rev. B 563875

[55] Frantsuzov P A, Volkan-Kacso S and Janko B 2013 Nano Lett. 13402

[56] Frantsuzov P, Kuno M, Jánko B and Marcus R A 2008 Nat. Phys. 4519

[57] Early, K T, McCarthy K D, Hammer N I, Odoi M Y, Tangirala R, Emrick T and Barnes M D 2007 Nanotechnology 18424027

[58] Zhang K, Chang H, Fu A, Alivisatos A P and Yang H 2006 Nano Lett. 6843

[59] Galland C, Ghosh Y, Steinbrück A, Sykora M, Hollingsworth J A, Klimov V I and Htoon H 2011 Nature 479 203

[60] Zhao J, Nair G, Fisher B R and Bawendi M G 2010 Phys. Rev. Lett. 104157403

[61] Rosen S, Schwartz O and Oron D 2010 Phys. Rev. Lett. 104157404

[62] Krauss T D and Brus L E 1999 Phys. Rev. Lett. 834840

[63] Rostron R J, Chao Y, Roberts G and Horrocks B R 2009 J. Phys. CM 21235301

[64] Cichos F, Martin J and von Borczyskowski C 2004 Phys. Rev. B 70115314

[65] Chao Y, Krishnamurthy S, Montalti M, Lie L H, Houlton A, Horrocks B R, Kjeldgaard L, Dhanak V R, Hunt M R C and Šiller L 2005 J. Appl. Phys. 98044316

[66] Yin J, Yue C, Zang Y, Chiu C-H, Li J, Kuo H-C, Wu Z, Li J, Fanga Y and Chen C 2013 Nanoscale 5, 4436

[67] Lie L H, Duerdin M, Tuite E M, Houlton A and Horrocks B R 2002 J. Electroanal. Chem. 538-539 183

[68] Žídek K, Pelant I, Trojánek F, Malý P, Gilliot P, Hönerlage B, Oberlé J, Šiller L, Little R and Horrocks B R 2011 Phys. Rev. B 84085321

[69] Chao Y, Šiller L, Krishnamurthy S, Coxon P R, Bangert U, Gass M, Kjeldgaard L, Patole S N, Lie L H, O'Farrell N, Alsop T A, Houlton A and Horrocks B R 2007 Nat. Nanotech. 2486

[70] Bhaduri G A, Little R, Khomane R B, Lokhande S U, Kulkarni B D, Mendis B G and Šiller L $2013 J$. Photochem. Photobiol. 2581

[71] Turkevich J, Stevenson P C and Hillier J 1951 Faraday Discuss. 1155

[72] Link S, Wang Z L and El-Sayed M A 1999 J. Phys. Chem. B 1033529

[73] Mishra P and Jain K P 2001 Phys. Rev. B 64073304 
[74] Hager J S, Zahardis J, Pagni R M, Compton R N and Li J 2004 J. Chem. Phys. 120, 2708

[75] Barnett S M, Harris N and Baumberg J J 2014 Phys. Chem. Chem. Phys. 166544

[76] Stiles P L, Dieringer J A, Shah N C and Van Duyne R P 2008 Annu. Rev. Anal. Chem. 1601

[77] Trojánek A, Langmaier J, Šebera J, Záliš S, Barbe J-M, Girault H H and Samec Z 2011 Chem. Commun. 47 5446

[78] Chung I and Bawendi M G 2004 Phys. Rev. B 70165304

[79] van Driel A F, Nikolaev I S, Vergeer P, Lodahl P, Vanmaekelbergh D and Vos W L 2007 Phys. Rev. B 75 035329

[80] Albery W J, Bartlett P N, Wilde C P and Darwent J R 1985 J. Am. Chem. Soc. 107, 1854 
Supporting information for 'Metal-enhanced luminescence of silicon quantum dots: effects of nanoparticles and molecular electron donors \& acceptors on the photofading kinetics'

\author{
Khamael M. Abualnaja ${ }^{1}$, Lidija Šiller ${ }^{1}$ and Benjamin R. Horrocks ${ }^{2 *}$ \\ ${ }^{1}$ School of Chemical Engineering and Advanced Materials, Herschel Building, Newcastle University, NE1 \\ 7RU, UK \\ ${ }^{2}$ Chemical Nanoscience Laboratory, School of Chemistry, Bedson Building, Newcastle University, NE1 7RU, \\ UK
}

\title{
Contents
}

1 Characterisation of alkylated silicon quantum dots $\left(\mathrm{C}_{11} \mathrm{SiQDs}\right) \quad 2$

2 Characterisation of AuNPs 3

3 Control data - Raman spectra for ferrocene and $\mathrm{FeCl}_{3}$ in the absence of SiQDs 5

*b.r.horrocks@ncl.ac.uk 


\section{Characterisation of alkylated silicon quantum dots $\left(\mathrm{C}_{11} \mathrm{SiQDs}\right)$}
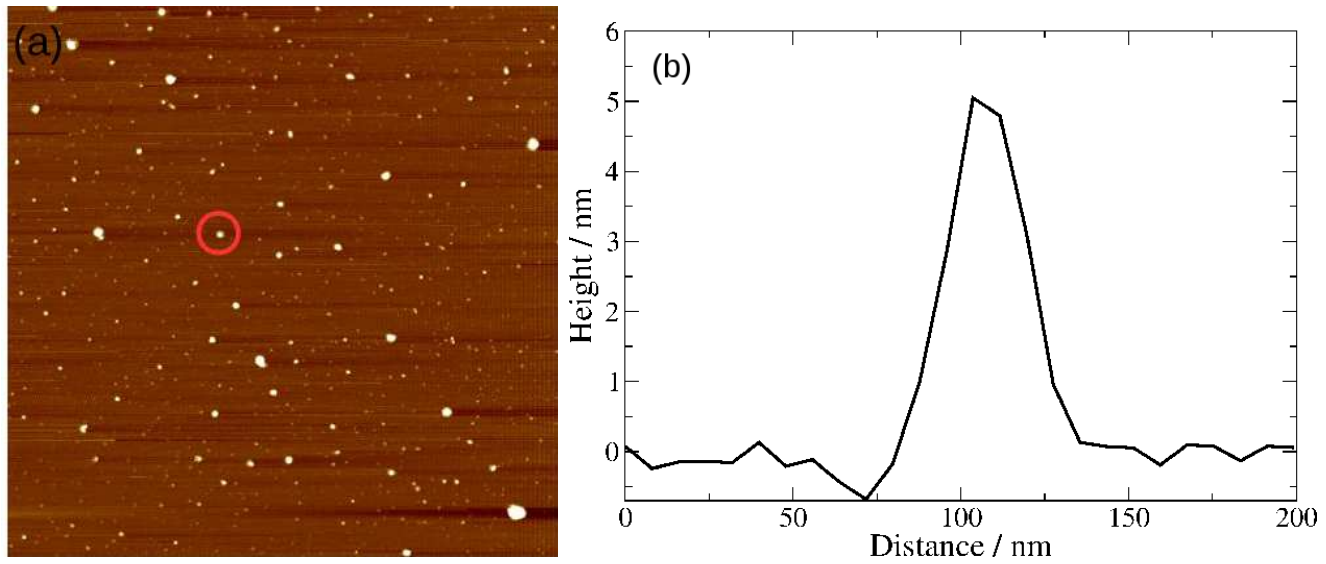

Figure 1: AFM image of $\mathrm{C}_{11} \mathrm{SiQDs}$ dried on a cleaved mica surface and imaged in tapping mode. (a) Height image 4 x 4 microns and the grayscale corresponds to $10 \mathrm{~nm}$; (b) Line section through one of the particles visible on the image (a) and indicated by a red circle.

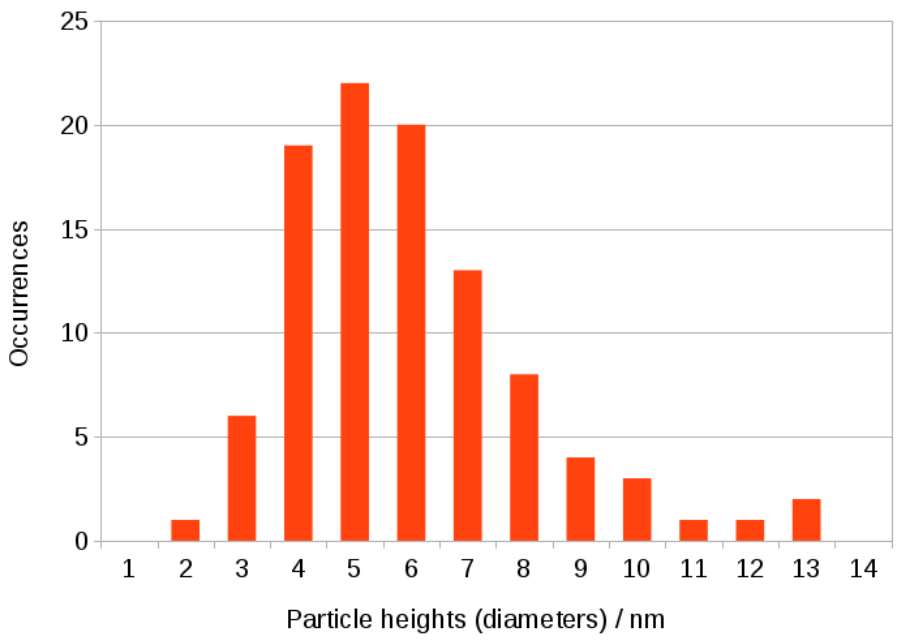

Figure 2: Histogram of particle heights (diameters) determined from AFM images. The mean particle diameter was $5.4 \mathrm{~nm}$ and the standard deviation was $2.1 \mathrm{~nm}$.

Figures (1) and (2) show an AFM image and a histogram of particle heights for a sample of $\mathrm{C}_{11}$-SiQDs dropcoated on a mica surface. We use the heights of the features to estimate the SiQD diameter (including the $\mathrm{C}_{11}$ alkyl capping monolayer) and find a mean diameter of $5.4 \pm 2.1 \mathrm{~nm}$ (mean \pm st. dev.). The histogram was compiled from 100 particles and 3 AFM images. The heights of the largest objects visible in figure (1) were still treated as estimates of individual particle diameters because these SiQDs are known to form islands [1]. 


\begin{tabular}{|c|c|}
\hline Assignment & Wavenumber $/ \mathrm{cm}^{-1}$ \\
\hline \hline $\mathrm{CH}_{3}$ methyl stretching vibration & 2956 \\
\hline $\mathrm{CH}_{2}$ asymmetric methylene stretch & 2926 \\
\hline $\mathrm{CH}_{2}$ symmetric methylene stretch & 2854 \\
\hline $\mathrm{CH}_{2}$ scissoring mode & 1459 \\
\hline $\mathrm{Si}-\mathrm{CH}_{2}-\mathrm{R}$ modes & 1259 \\
\hline $\mathrm{Si}-\mathrm{O}-\mathrm{Si}$ (broad, weak) & 1050 \\
\hline $\mathrm{C}-\mathrm{H}$ bending & 615 \\
\hline
\end{tabular}

Table 1: Band assignments for FTIR spectra of $\mathrm{C}_{11}$-SiQDs.

FTIR spectral data for the $\mathrm{C}_{11}$-SiQDs. A detailed discussion of these spectra has been reported in Refs $[1,2]$. Si-Si modes are of course not observed in FTIR absorption spectra, but are seen in the Raman spectra presented in the main text.

\section{Characterisation of AuNPs}

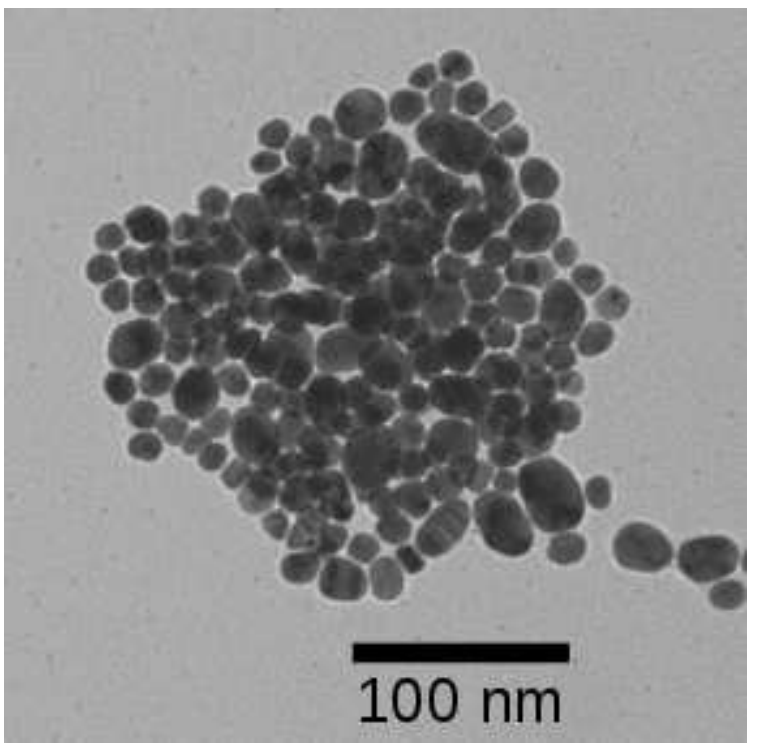

Figure 3: Transmission electron microscopy of AuNPs. Magnification $\times 130,000$.

Transmission electron micrograph of the AuNPs employed. The mean particle diameter was $20 \mathrm{~nm}$ and the range of sizes was from $13-30 \mathrm{~nm}$. 


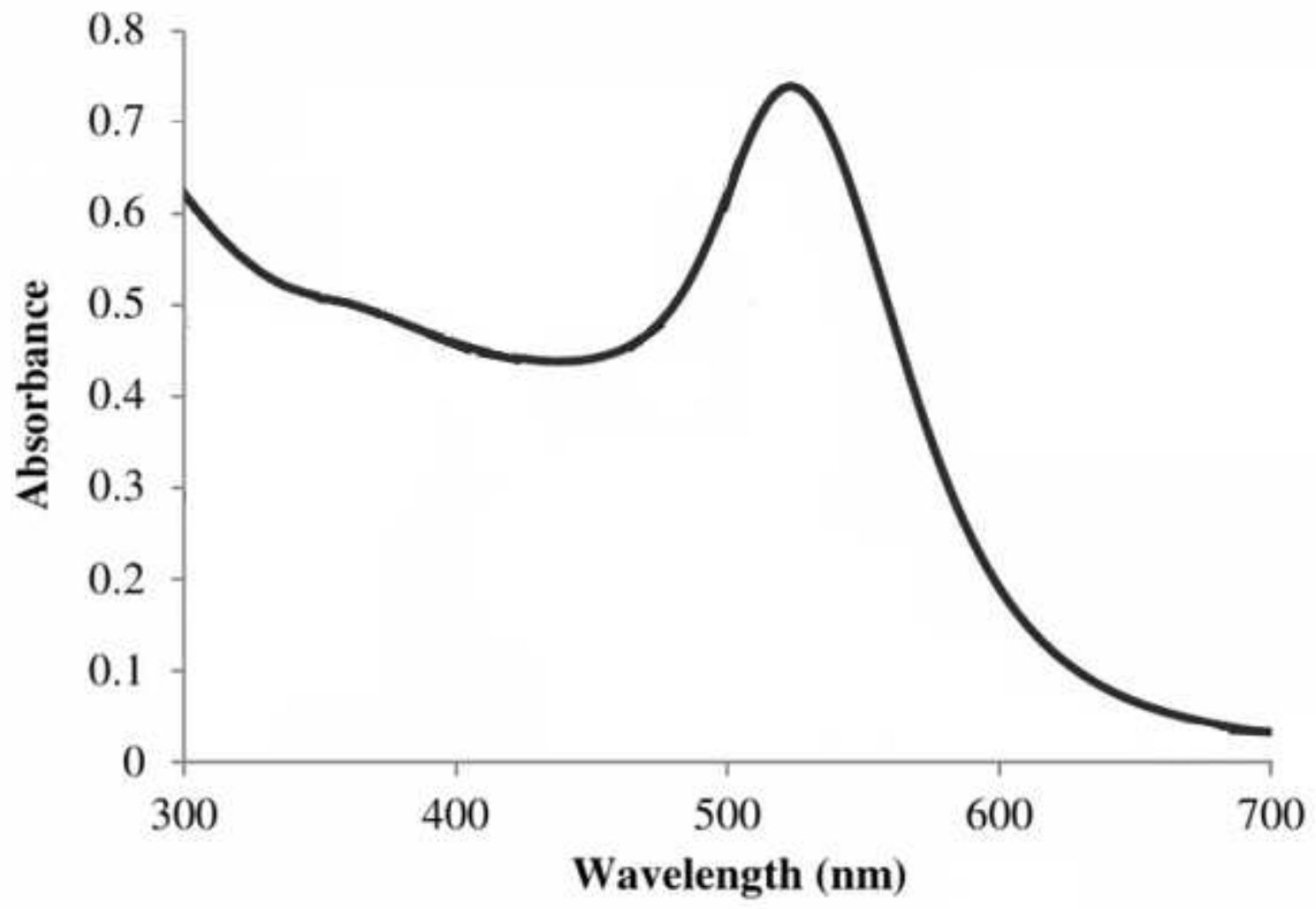

Figure 4: UV-Vis absorption spectrum of the as-prepared aqueous suspension of AuNPs.

Optical absorption spectrum of the AuNPs in aqueous suspension. The peak near $520 \mathrm{~nm}$ is the plasmon absorption expected for Au NPs [3, 4]. 
3 Control data - Raman spectra for ferrocene and $\mathrm{FeCl}_{3}$ in the absence of SiQDs

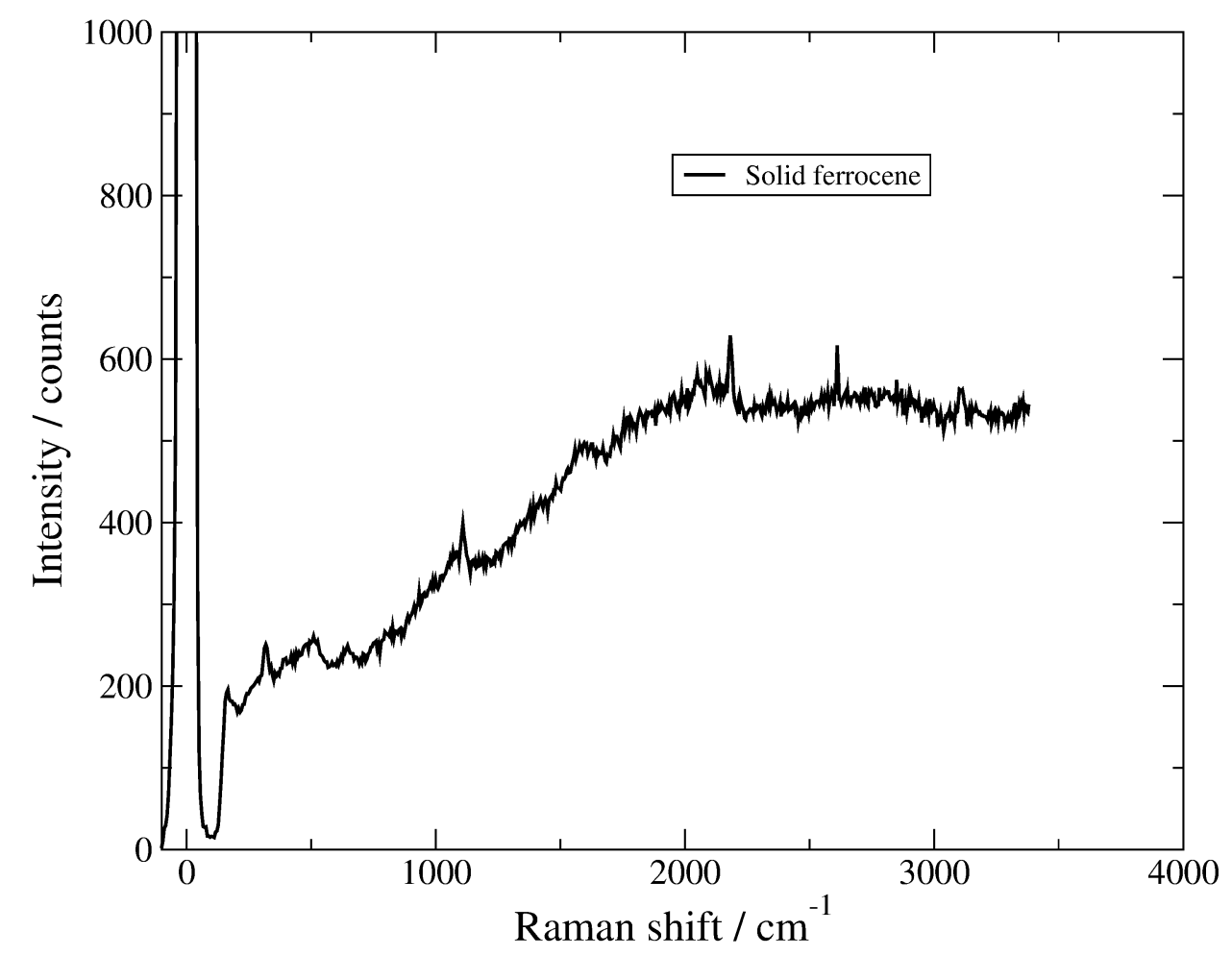

Figure 5: Raman-luminescence spectrum of solid ferrocene. The laser wavelength was $488 \mathrm{~nm}$ and the integration time was 60 s. 


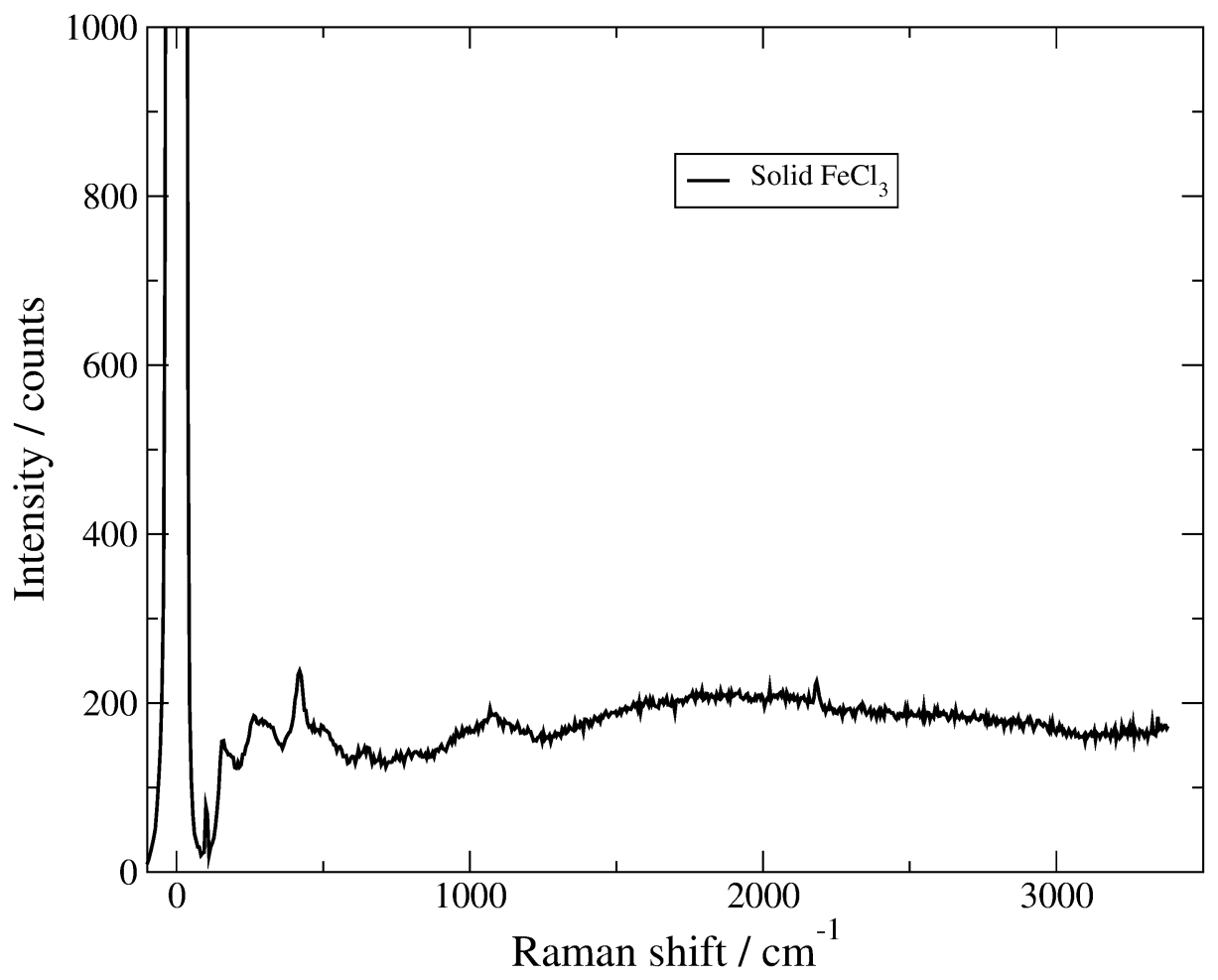

Figure 6: Raman-luminescence spectrum of solid $\mathrm{FeCl}_{3}$. The laser wavelength was $488 \mathrm{~nm}$ and the integration time was 60 s.

\section{References}

[1] Chao Y, Šiller L, Krishnamurthy S, Coxon P R, Bangert U, Gass M, Kjeldgaard L, Patole S N, Lie L H, O'Farrell N, Alsop T A, Houlton A and Horrocks B R 2007 Nat. Nanotech. 2486.

[2] Lie L H, Duerdin M, Tuite E M, Houlton A and Horrocks B R 2002 J. Electroanal. Chem. 538-539 183

[3] Turkevich J, Stevenson P C and Hillier J 1951 Faraday Discuss. 1155

[4] Link S, Wang Z L and El-Sayed M A 1999 J. Phys. Chem. B 1033529. 